\title{
Preventive Effects of Fermented Brown Rice and Rice Bran on Spontaneous Lymphomagenesis in AKR/NSlc Female Mice
}

\author{
Toshiya Kuno ${ }^{1}$, Hiroyuki Kato ${ }^{1}$, Aya Naiki-Ito ${ }^{1}$, Shugo Suzuki ${ }^{1}$, Takuji Tanaka ${ }^{2 *}$, \\ Satoru Takahashi ${ }^{1}$, Hideki Mori ${ }^{3}$
}

\begin{abstract}
Fermented brown rice and rice bran with Aspergillus oryzae (FBRA) is known to possess potentials to prevent chemical carcinogenesis in multiple organs of rodents. In the present study, possible chemopreventive effect of FBRA against spontaneous occurrence of lymphomas was examined using female AKR/NSlc mice. Four-week-old female AKR/ NSlc mice were divided into three groups, and fed diets containing FBRA for 26 weeks at a dose level $0 \%$ (Group 1), 5\% (Group 2) or 10\% (Group 3). At the termination of experiment, the incidence of thymic malignant lymphoma of Group 3 was significantly lower than of Group $1(p<0.05)$. The average number of apoptotic cells of the thymic lymphoma of Group 3 was significantly larger than that of Group $1(\mathrm{p}<0.05)$. In addition, the incidences of malignant lymphoma arising from body surface and abdominal lymph nodes, and the frequencies of lymphoma cell invasion to liver, kidney, spleen, and ovary of Group 3 were relatively lower than those of Group 1. These results indicate that FBRA inhibits spontaneous development of the lymphoma in female AKR/NSc mice and the inhibition of lymphomagenesis may relate to the induction of apoptosis by exposure of FBRA, suggesting that FBRA could be a protective agent against development of human lymphoma.
\end{abstract}

Keywords: Fermented brown rice and rice bran- spontaneous malignant lymphoma- chemoprevention- Mice

Asian Pac J Cancer Prev, 19 (11), 3217-3223

\section{Introduction}

The number of patients with lymphoma is reportedly increasing worldwide and approximately 300,000 new cases of lymphoma develop per year (Medeiros et al., 2017). It is known that the incidence of lymphoma varies according to the specific type and the geographic location in the world, and the variation is likely attributable to both genetic and socioeconomic factors (Medeiros et al., 2017). In Japan, the center for cancer control and information services of the National Cancer Center reported malignant lymphoma incidences of 22.3/100,000 and $18.3 / 100,000$ in men and women, respectively, in 2013 (Miyoshi and Ohshima, 2018).

Brown rice is a staple dietary constituent in Asia, whereas rice consumed in the Western countries is generally white rice obtained from brown rice by removal of the bran. Fermented brown rice and rice bran with Aspergillus (A) oryzae (FBRA) is a food manufactured by fermenting a mixture of brown rice and rice bran with $\mathrm{A}$. oryzae to improve its digestibility (Henderson et al., 2012). As reviewed by Henderson et al.
(Henderson et al., 2012), FBRA exerts its chemopreventive effects against chemical carcinogenesis in different tissues, including colon (Katayama et al., 2002), liver (Katayama et al., 2003), esophagus (Kuno et al., 2004), urinary bladder (Kuno et al., 2006), stomach (Tomita et al., 2008), lung (Phutthaphadoong et al., 2009), pancreas (Kuno et al., 2015) of rodents. Recently, this dietary agent was found to be protective on spontaneous induction of prostate tumorigenesis in transgenic rats developing adenocarcinoma of the prostate (TRAP) bearing the SV40 T antigen transgene under control of the probasin promoter (Kuno et al., 2016). To our knowledge, no successful studies regarding chemoprevention of haematopoietic and lymphoid tissues by dietary rice bran and FBRA have been reported.

The AKR mouse strain is characterized by a high incidence of spontaneous thymic lymphoma that appears in older animals and is associated with novel provirus integrations of ectopic and recombinant murine leukemia viruses (Richie et al., 1991). The present study was undertaken to examine effect of FBRA on the spontaneous lymphoma developed in female 
AKR mice (Pattengale and Taylor, 1983). To invest mode of action of FBRA on the lymphomagenesis, assay of apoptosis emerged in the lymphoma cells was performed.

\section{Materials and Methods}

\section{Animals}

Female AKR/NSlc mice, 4 weeks old $(\mathrm{n}=75)$ were purchased from Japan SLC, Inc (Hamamatsu, Japan). All animals were housed in plastic cages on wood-chip bedding in an air-conditioned specific pathogen-free animal room at $22 \pm 2{ }^{\circ} \mathrm{C}$ and $55 \pm 5 \%$ humidity with a $12 \mathrm{~h}$ light/dark cycle. The animals were fed a basal diet (Oriental MF, Oriental Yeast, Tokyo, Japan) and provided with water ad libitum. All animals were performed under protocols approved by the Institutional Animal Care and Use Committee of Nagoya City University Graduate School of Medical Sciences. The mice were divided into 3 groups of 25 mice each. FBRA was obtained from Genmai Koso Co. Ltd. (Sapporo, Japan) and freshly prepared for administration to the treatment groups. The manufacturing process for FBRA was indicated in our previous report (Kuno et al., 2015). The process contains two steps of fermentation. The 1st step is to produce a fermentation base by steaming brown rice and rice bran. Then $A$. oryzae is seeded into the fermentation base. This fermentation process is continued for $1824 \mathrm{~h}$ and the 2 nd fermentation is continued for an additional $1224 \mathrm{~h}$ for aging purposes. The fermented product is finally dried and powdered. The experimental diets were prepared by mixing FBRA, which were reported as effective concentrations (Katayama et al., 2002; Kuno et al., 2004; Tomita et al., 2008; Phutthaphadoong et al., 2009) with the control diet.

\section{Experimental procedure}

The animals were fed a diet containing $0 \%$ (Group 1), $5 \%$ (Group 2) or 10\% (Group 3) FBRA for 26 weeks, and body weight and food consumption were measured weekly. At the end of experiment all surviving animals were sacrificed under deep anesthesia by deep inhalation of isoflurane and blood samples from selected mice Groups $1(\mathrm{n}=19)$, $2(n=19)$ and $3(n=18)$ were collected for determining serum lactate dehydrogenase (LDH) from the aorta after overnight fasting. Serum LDH was measured by SRL, Inc., Tokyo, Japan. At necropsy, body surface lymph nodes, thymus, abdominal lymph nodes, liver, kidney, and ovary were carefully examined macroscopically. All tissues from these organs were fixed in $10 \%$ buffered formalin. All tissues were routinely processed, embedded in paraffin, sectioned to a thickness of $4 \mu \mathrm{m}$, and stained with hematoxylin and eosin (H \& E) to assess histological features.

\section{Immunohistochemistry}

Deparaffinized sectioned were incubated with diluted antibodies. Activity for cell proliferation was detected by use of anti-Ki-67 antibody (Abcam, Cambridge, UK, \#ab1667, 1:100). Apoptopic cells were detected by terminal deoxy nucleotidyl transferase-mediated dUTP nick end labeling (TUNEL) assay using an in situ
Apoptosis Detection Kit from Takara Bio Inc. (Otsu, Japan). The labeling indices of TUNEL were determined by counting at least 1000 lymphoma cells under a microscope light microscopy at high magnification.

\section{Western Blot Analysis}

The harvested frozen tissues from the thymic lymphoma were homogenized in radioimmunoprecipitation assay buffer containing a protease inhibitor (1mM phenylmethylsulfonyl fluoride). Total cellular proteins were quantified by the Bradford procedure and equal amounts of proteins were mixed with Laemmli sample buffer (Bio-Rad Laboratories, Inc, , Hercules, CA, USA) and fractionated by gel electrophoresis in $6 \%$ or $12 \%$ polyacrylamide resolving gels containing $0.1 \%$ SDS. Proteins were transferred onto Hybond ECL nitrocellulose membranes (Amersham Biosciences, Little Chalfont, UK), which were subsequently incubated overnight with primary antibodies against cleaved caspase 3 (CST, \#9661, 1:1000), cleaved caspase 7 (CST, \#9491, 1:1,000), and PARP (CST, \#9542, 1:1,000). Equal protein loading was ascertained by western blotting with an anti- $\beta$-actin antibody (Sigma-Aldrich, St. Louis, MO, USA, \#A5316, 1:5,000). The signal was detected using an ImageQuant LAS-4000 mini biomolecular imager (GE Healthcare, Little Chalfont, UK). The intensity of each band was measured using Image J 1.48v (National Institutes of Health, Bethesda, MD, USA).

\section{Statistical Analysis}

The data expressed as mean \pm standard deviation (SD) were analyzed using one-way analysis of variance followed by Dunnett's test. The incidences of lesions between the groups were expressed compared by two tailed Chi-square test without Yates' correction. Differences between the groups were considered to be significant at $\mathrm{p}<0.05$. All statistical analyses were performed using GraphPad Prism software (version 6; GraphPad Software, Inc., La Jolla, USA).

\section{Results}

There were not clear differences on the food intake/day among the groups throughout the experimental period (Figure 1a). No clear differences were also noted for the process of body weight gain among the groups during the experiment (Figure 1b). At the end of the study, mean body weights, and weights of several organs (liver, kidney, and spleen) of Groups 2 (body weight, $39.0 \pm 7.6 \mathrm{~g}$; liver weight, $1.8 \pm 0.3 \mathrm{~g}$; bilateral kidney weight, $0.41 \pm 0.06 \mathrm{~g}$; spleen weight, $0.14 \pm 0.11 \mathrm{~g}$ ) and 3 (body weight, $38.6 \pm 6.4 \mathrm{~g}$; liver weight, $1.7 \pm 0.2 \mathrm{~g}$; bilateral kidney weight, $0.41 \pm 0.04$ $\mathrm{g}$; spleen weight, $0.13 \pm 0.13 \mathrm{~g}$ ) were slightly smaller than those of Group 1 (body weight, $40.3 \pm 7.0 \mathrm{~g}$; liver weight, $2.0 \pm 0.6 \mathrm{~g}$; bilateral kidney weight, $0.42 \pm 0.05 \mathrm{~g}$; spleen weight, $0.25 \pm 0.39 \mathrm{~g}$ ). However, no significant differences were confirmed. The mean weight of thymus $(0.18 \pm 0.18 \mathrm{~g})$ in Group 3, but not Group $2(0.31 \pm 0.44 \mathrm{~g})$, was smaller than that of Group $1(0.26 \pm 0.24 \mathrm{~g})$ without statistically significance. There were no clear histological signs of toxicity related to FBRA intake in the liver and kidney of 
(a)

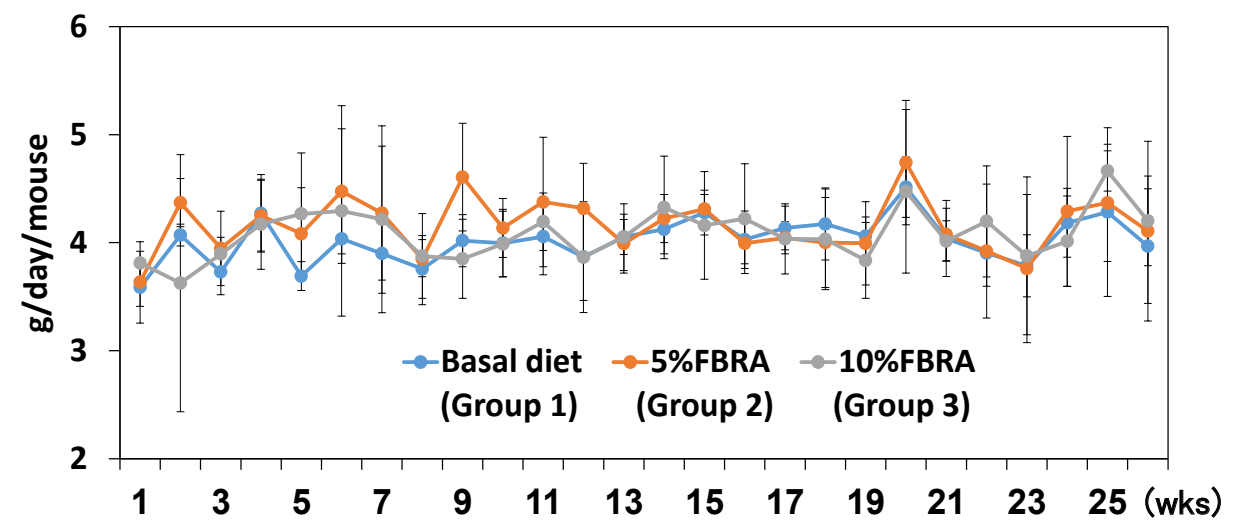

Experimental period

(b)

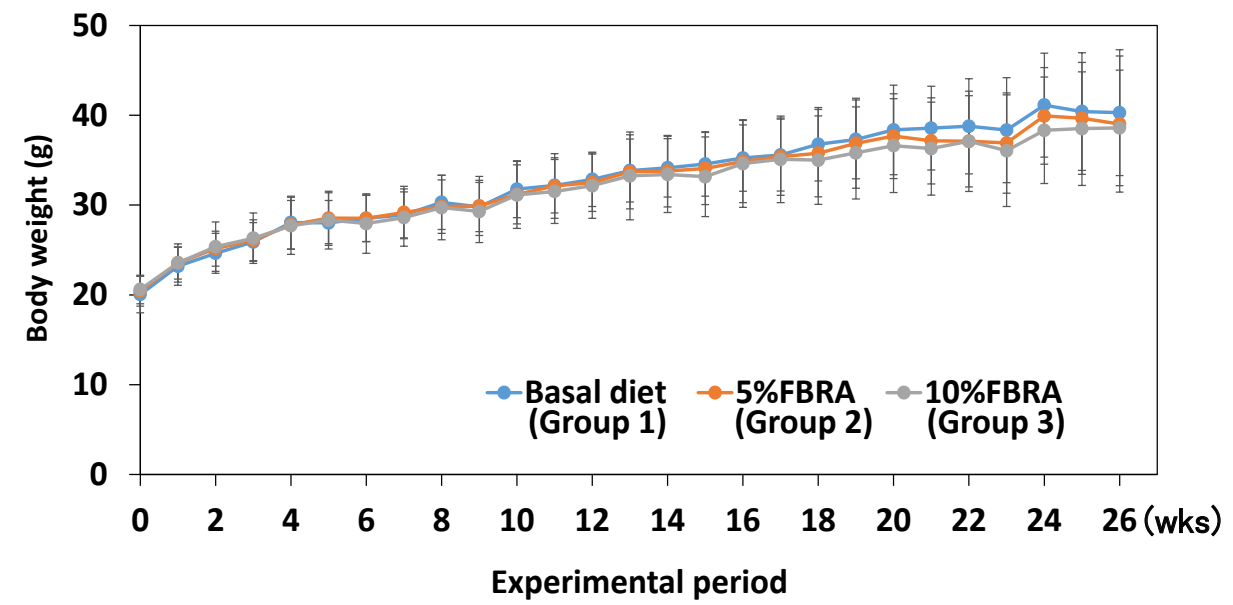

Figure 1. Mean Food Intakes (a) and Mean Weight Gains (b) of All Groups During the Experiments. No significant differences on both parameters among the group are noted..

the mice belonging to Groups 2 and 3 (data not shown). The mean serum LDH value of Group $3(n=18)$ was lower than of Group $1(n=19)$ or Group $2(n=19)$ at the end of the study (Figure 2), but the differences were statistically insignificant. At the sacrifice, mice particularly of Group 1 had strongly swelled thymus, hepatosplenomegaly, and swelled abdominal lymph nodes (Figure 3 ).
Histopathologically, the enlarged thymus was replaced by diffuse growth and invasion of atypical lymphocytes with starry-sky appearance (Figure 4a) and frequent mitosis and apoptosis (Figure 4b). Immunohistochemically, neoplastic cells were positive for CD3 (Figure 4c) and negative for CD20 (Figurre 4d). Thus, the neoplasms developed in the thymus were diagnosed as lymphoblastic

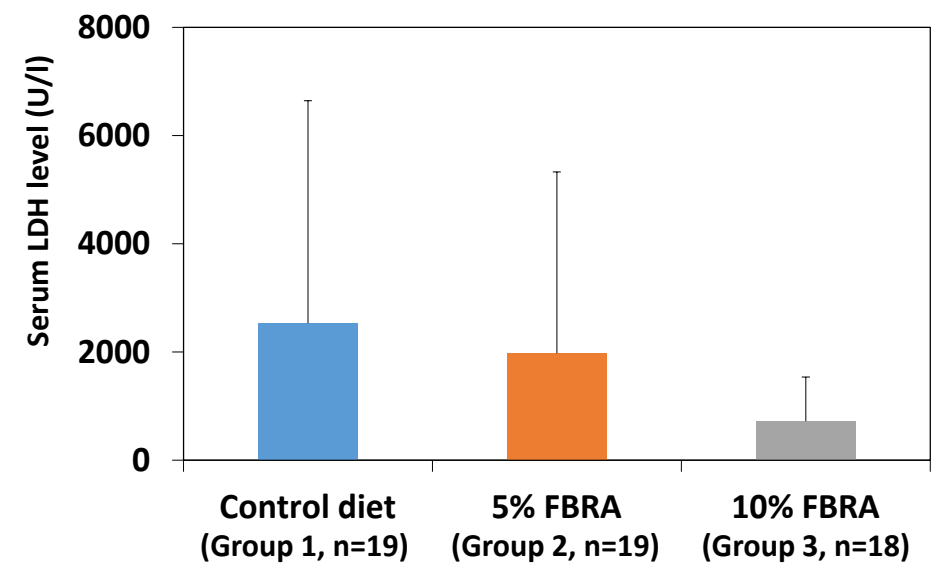

Figure 2. Mean Values of Serum LDH of All Groups. The values of mice fed FBRA at a dose level of 5\% (Group 2) and 10\% (Group 3) are lower than that of mice fed control diet without FBRA (Group 1), but the differences are insignificant. 
Table 1. Incidence of Malignant Lymphoma and Tissues where Lymphoma Cells Involved

\begin{tabular}{|c|c|c|c|c|c|c|c|c|}
\hline \multirow[t]{2}{*}{ Group no. (Treatment) } & \multirow[t]{2}{*}{ No. of mice examined ${ }^{*}$} & \multicolumn{3}{|c|}{ No. of mice with lymphoma (\%) } & \multicolumn{4}{|c|}{$\begin{array}{c}\text { Tissues where lymphoma cells } \\
\text { infiltrated (\%) }\end{array}$} \\
\hline & & Thymus & Surface lymph node & Abdominal lymph nodes & Liver & Kidney & Spleen & Ovary \\
\hline Group 1 (Control diet) & 25 & $15(60)$ & $8(32)$ & $7(28)$ & $8(32)$ & $7(28)$ & $9(36)$ & $5(20)$ \\
\hline Group 2 (5\% FBRA) & 23 & $14(61)$ & $6(26)$ & $6(26)$ & $6(26)$ & $5(22)$ & $7(30)$ & $4(17)$ \\
\hline Group 3 (10\% FBRA) & 24 & $7(29)^{* *}$ & $4(17)$ & $4(17)$ & $4(17)$ & $3(13)$ & $4(17)$ & $3(13)$ \\
\hline
\end{tabular}

*No. of mice survived at the end of study. ** $\mathrm{p}<0.05$, vs. Group 1 (control diet).

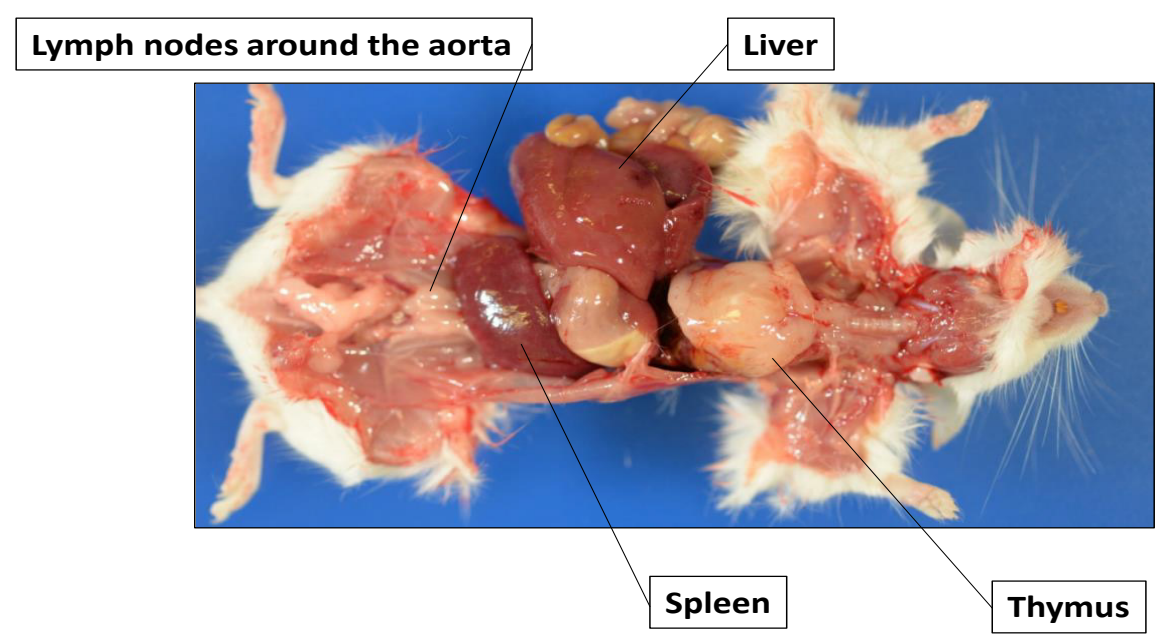

Figure 3. Representative Macroscopic View of an Autopsy Case of a Mouse Belonging to Group 1. Note enlarged thymus, hepatospelenomegaly, and enlarged abdominal lymph nodes around the aorta.

T cell lymphoma. Table 1 summarizes the incidences of lymphomas in different tissues and those of the tissues where lymphoma cells infiltrated (Figure 5). As evaluated by the Ki-67 assay, lymphomas of all groups had very high cell proliferating activity $(>90 \%)$, thereby no differences were noted among the groups (data not shown). Figure 6 shows the mean number of apoptotic cells (/high power field) of the lymphomas developed in all groups. The mean number of apoptosis in Group 3 (10\% FBRA) was significantly higher than that of the Group $1(\mathrm{p}<0.01)$. Immunoblot analysis proved activation of cleaved caspase 3 , cleaved caspase 7 , and PARP in the lymphomas of all groups (Figure 7). Expression of three proteins associated apoptosis in lymphoma developed in Groups 2 and 3 was relatively greater than that of Group 1. FBRA feeding did not affect

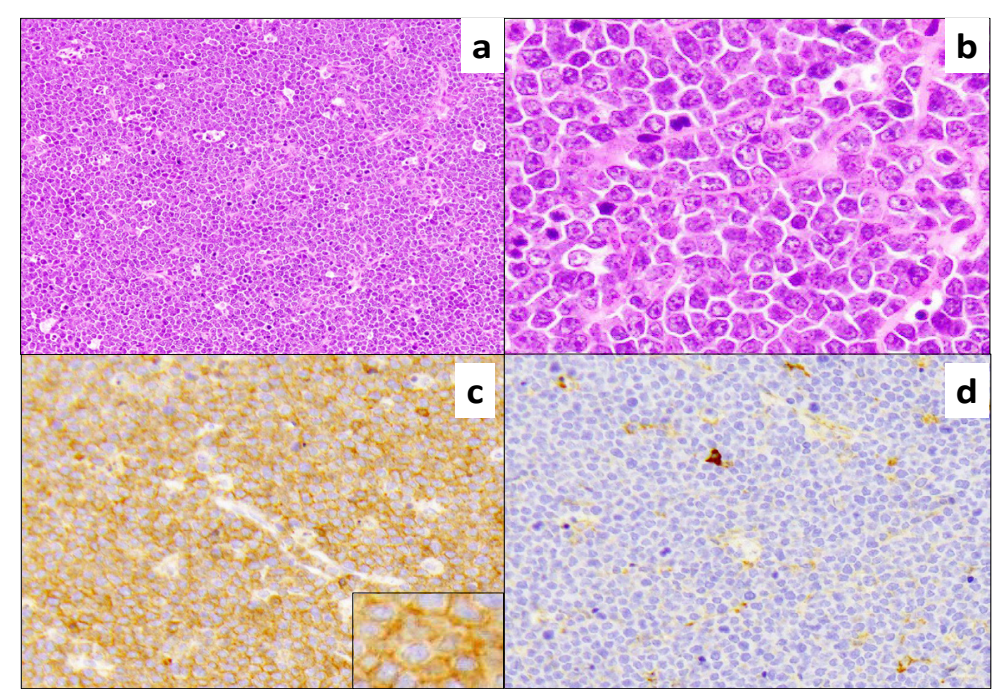

Figure 4. Representative Histopathology and Immunohistochemistry of Thymic Lymphoma Developed in a Mouse of Group 1. Note (a) the growth of atypical lymphocytes with Starry-sky appearance in the thymus and (b) many nuclear mitoses and apoptotic lymphoma cells. Lymphoma cells show immunohistochemically positive reaction against (c) CD3 and negative against (d) CD20. (a) and (b), H \& E stain and (c) and (d) immunohistochemistry. Original magnification, (a) x200, (b) x1000, (c) x400, (d) x400. Insert in (c), x1000. 


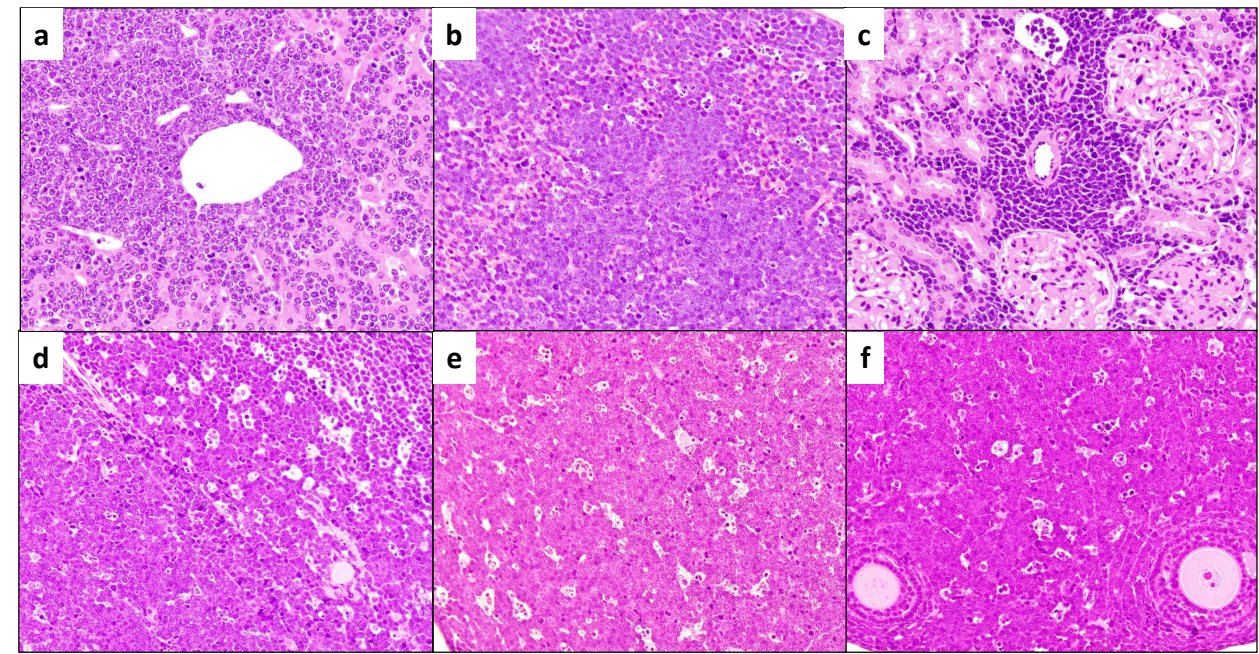

Figure 5. Representative Histopathology and of Tissues where Lymphoma Cells Infiltrated in a Mouse of Group 1. (a) liver, (b) spleen, (c) kidney, (d) cervical lymph node, (e) abdominal lymph node and (f) ovary. H \& E stain, Original magnification, $\mathrm{x} 400$.
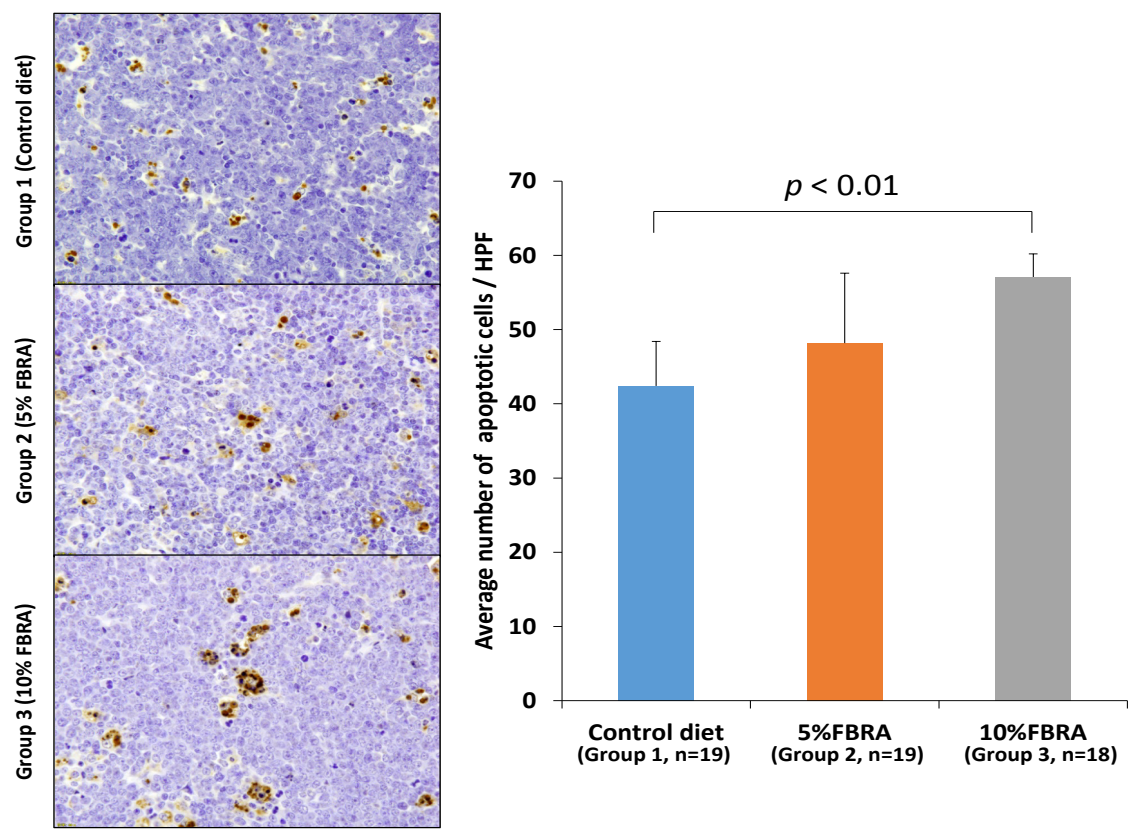

Figure 6. Apoptosis in the Thymic Lymphoma. TUNEL assay shows representative apoptotic cell nuclei (stained in brown, left column). The mean numbers of apoptotic cells (/HPF) in thymic lymphomas developed in all groups reveled that the value of Group $3(10 \%$ FBRA) is significantly larger than that of Group 1 ( $p<0.01$, right column). Left colomn: TUNEL immunohistochemistry, Original magnificantion x1000.

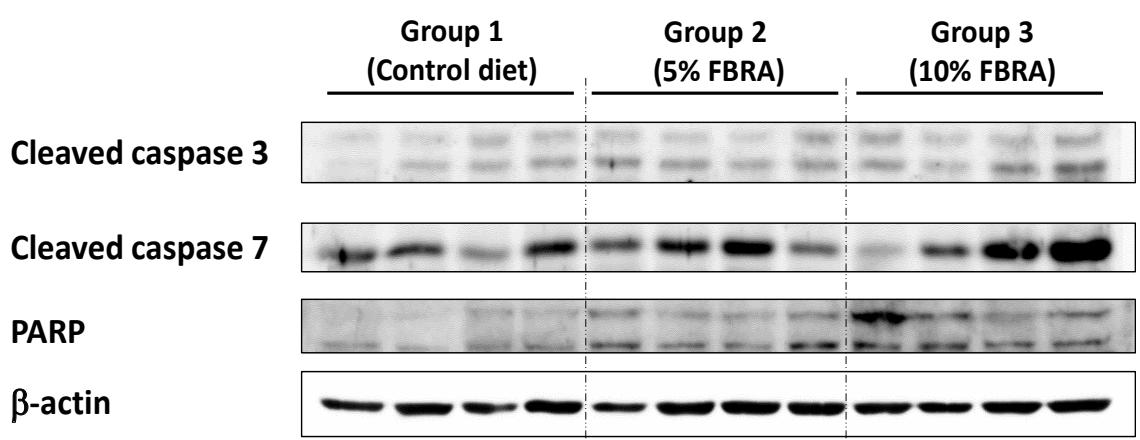

Figure 7. Representative Images of Western Blot Analysis Investigating Expression of Apoptosis-related Proteins, Cleaved Caspase 3, Cleaved Caspase 7 and PARP in Thymic lymphomas Developed in Groups 1 Through 3. Activation of cleaved caspase 3 and cleaved caspase 7, and PARP in Groups 2 and 3 was slightly greater than that of Group 1. 
the apoptosis in non-neoplastic cells in the thymus and/ or in thymic lymphoma cells infiltrated into tissues, such as liver (data not shown).

\section{Discussion}

Recent epidemiological analysis indicates a clear association with socioeconomic status regarding the incidence of lymphomas; they are more common in developed nations than developing countries. These factors likely explain the high incidence of lymphomas in the United States and other industrialized nations (Medeiros et al., 2017). Concerning to epidemiologic risk factors for non-Hodgkin's lymphomas, heredity, infectious agents, immunosuppression, and autoimmune disease are regarded as established factors, and genetic polymorphisms, exposure to agricultural agents, hair dyes, and immunomodulator therapies are regarded as suggestive risk factors. Diet, alcohol use, and tobacco use are regarded as not established risk factors (Medeiros et al., 2017). However, there is a recent experimental study reporting that diet-induced obesity accelerates acute lymphoblastic leukemia progression in two murine models including AKR mice (Yun et al., 2010). Furthermore, it is also reported that long-term effects of curcumin prevented progression of Dalton's T cell lymphoma, which was intraperitoneally transplated in the AKR mice, by inducing phase-II antioxidant enzymes via activation of Nrf2 signaling, restoration of $\mathrm{p} 53$, and modulation of inflammatory mediators, such as tumor growth factor- $\beta$ and cyclooxygenase- 2 in liver (Das and Vinayak, 2015). These evidences suggest that diet may be a risk factor or a preventive agent for lymphomas. Indeed several natural or synthetic products (Menezes et al., 2016; Aregueta-Robles et al., 2018; Ye et al., 2018) are reported to reduce the risk of certain types of lymphoma.

In this study, the incidence of thymic lymphoma appeared in the mice fed the diet containing $10 \%$ FBRA for 26 weeks (Group 3) was significantly lower than that of the group given the control diet alone ( $0 \%$ FBRA, Group 1), indicating the preventive effects of FBRA on the spontaneous lymphomagenesis in the female AKR mice. Underlying mechanism of the prevention of FBRA on the development of the lymphoma is not known. However, in the current study, dietary exposure of $10 \%$ FBRA enhanced emergence of apoptosis in the lymphoma cells. Recently, Horie et al., (Horie et al., 2016) reported that fermented brown rice causes apoptotic death in human acute lymphoblastic leukemia cells via death receptor pathway and supplementarily through the tBid-mediated mitochondrial pathway. Accordingly, induction of apoptosis may be important for the preventive effect on the lymphomagenesis. Certainly, it is also suggested that dietary exposure of FBRA induces cellular apoptosis in the preneoplastic and neoplastic stages of lymphomagenesis, thereby elongates the latent period of development of the lymphoma in the mice.

Conflict of interest statement

The authors declare that they have nothing to disclose.

\section{References}

Aregueta-Robles U, Fajardo-Ramirez OR, Villela L, et al (2018). Cytotoxic activity of a black Bean (Phaseolus vulgaris L.) extract and its flavonoid fraction in both in vitro and in vivo models of lymphoma. Rev Invest Clin, 70, 32-9.

Das L, Vinayak M (2015). Long term effect of curcumin in restoration of tumour suppressor p53 and phase-II antioxidant enzymes via activation of $\mathrm{Nrf} 2$ signalling and modulation of inflammation in prevention of cancer. PLoS One, 10, e0124000.

Henderson AJ, Ollila CA, Kumar A, et al (2012). Chemopreventive properties of dietary rice bran: current status and future prospects. Adv Nutr, 3, 643-53.

Horie Y, Nemoto H, Itoh M, et al (2016). Fermented brown rice extract causes apoptotic death of human acute lymphoblastic Leukemia cells via death receptor pathway. Appl Biochem Biotechnol, 178, 1599-611.

Katayama M, Sugie S, Yoshimi N, et al (2003). Preventive effect of fermented brown rice and rice bran on diethylnitrosoamine and phenobarbital-induced hepatocarcinogenesis in male F344 rats. Oncol Rep, 10, 875-80.

Katayama M, Yoshimi N, Yamada Y, et al (2002). Preventive effect of fermented brown rice and rice bran against colon carcinogenesis in male F344 rats. Oncol Rep, 9, 817-22.

Kuno T, Hirose Y,HataK, etal(2004). Preventive effect of fermented brown rice and rice bran on N-nitrosomethylbenzylamineinduced esophageal tumorigenesis in rats. Int J Oncol, 25, 1809-15.

Kuno T, Hirose Y, Yamada Y, et al (2006). Chemoprevention of mouse urinary bladder carcinogenesis by fermented brown rice and rice bran. Oncol Rep, 15, 533-8.

Kuno T, Nagano A, Mori Y, et al (2016). Preventive effects of fermented brown rice and rice bran against prostate carcinogenesis in TRAP rats. Nutrients, 8, 421.

Kuno T, Takahashi S, Tomita H, et al (2015). Preventive effects of fermented brown rice and rice bran against $\mathrm{N}$-nitrosobis (2-oxopropyl) amine-induced pancreatic tumorigenesis in male hamsters. Oncol Lett, 10, 3377-84.

Medeiros LJ, O’Malley DP, Caraway NP, et al (2017). Epidemiology of Hodgkin and non-Hodgkin lymphomas. In 'Tumors of the Lymph nodes and spleen', Ed. Silverberg, S.G., American Registry of Pathology, Washington, DC, pp 119-28.

Menezes JC, Orlikova B, Morceau F, et al (2016). Natural and synthetic flavonoids: Structure-activity relationship and chemotherapeutic potential for the treatment of leukemia. Crit Rev Food Sci Nutr, 56, 4-28.

Miyoshi H, Ohshima K (2018). Epidemiology of malignant lymphoma and recent progress in research on adult T-cell leukemia/lymphoma in Japan. Int J Hematol, 107, 420-7.

Pattengale PK, Taylor CR (1983). Experimental models of lymphoproliferative disease. The mouse as a model for human non-Hodgkin's lymphomas and related leukemias. Am J Pathol, 113, 237-65.

Phutthaphadoong S, Yamada Y, Hirata A, et al (2009). Chemopreventive effects of fermented brown rice and rice bran against 4-(methylnitrosamino)-1-(3-pyridyl)-1butanone-induced lung tumorigenesis in female A/J mice. Oncol Rep, 21, 321-7.

Richie ER, Angel JM, Cloyd MW (1991). Influence of murine leukemia proviral integrations on development of N-methyl$\mathrm{N}$-nitrosourea-induced thymic lymphomas in AKR mice. $J$ Virol, 65, 5751-6.

Tomita H, Kuno T, Yamada Y, et al (2008). Preventive effect of fermented brown rice and rice bran on N-methyl-N'-nitro$\mathrm{N}$-nitrosoguanidine-induced gastric carcinogenesis in rats. 
Oncol Rep, 19, 11-5.

Ye X, Zhang G, Righolt C, et al (2018). Associations between statin use and risk of non-Hodgkin lymphomas by subtype. Int J Cancer, 143, 971-9.

Yun JP, Behan JW, Heisterkamp N, et al (2010). Diet-induced obesity accelerates acute lymphoblastic leukemia progression in two murine models. Cancer Prev Res (Phila), 3, 1259-64.

\section{c) (i) (8)}

This work is licensed under a Creative Commons AttributionNon Commercial 4.0 International License. 